\title{
A Novel Method of Resin-Embedding Thin, Flexible Polymer Films for TEM Sectioning
}

\author{
K. Ward*, M. Chun*, C. Queenan*, A. Calabro*, J. Morales**, D. Becker*
}

* Bergen County Academies, Nano-Structural Imaging Lab, 200 Hackensack Avenue, Hackensack, NJ 07601

** The City College of New York, Electron Microscopy Facility, 160 Convent Avenue, New York, NY10031

Coated thin polymer films are found in numerous food, drink and medical packaging systems and are used as a barrier to slow the migration of outside contaminants into the product, or the product out of the packaging. Structural visualization is critical to elucidating coating-polymer interactions and in examining the uniformity of coating in production of these films. One method used to analyze these films is cross-sectional TEM analysis. Previous publications have explained methods of preparing metallic substrates [1], solid thin films [2], and rigid multilayer polymers to coat items like camera lenses [3] for cross-sectional analysis. This paper will propose a new method for embedding and sectioning flexible, transparent polymer films that are shown here to be unsuitable for ultramicrotomy and imaging using current techniques.

Because the polymer films are thin and flexible (like a piece of saran wrap), normal embedding and cutting techniques do not provide the necessary support for the film to retain its shape. Images taken of the films using current techniques, such as embedding a single flat strip in resin or sandwiching multiple strips together prior to sectioning, showed signs of damage or folding of the film (Figure 1). The method that follows corrected the problems of the film folding or the edges tearing during the embedding and sectioning process.

A $2 \mathrm{~cm} \times 6 \mathrm{~cm}$ strip was cut from the sheet of film. The strip was carefully rolled around a wooden pick $(65 \mathrm{~mm} \times 2 \mathrm{~mm})$. Once tightly rolled, one end of the film was folded over (approximately $1 \mathrm{~cm}$ from the top) and taped, using standard laboratory labeling tape, to allow the film to retain its rigid tube shape. The opposite end was trimmed to ensure that the film edge was even. An epoxy resin (EMBed 812, Electron Microscopy Sciences) was prepared and pipetted into a BEEM capsule for each sample. The rolled film was then lowered into the center of the BEEM capsule using forceps. The samples were placed in a vacuum desiccator at $25 \mathrm{in}$ of $\mathrm{Hg}$ for 30 minutes and then moved into a $60^{\circ} \mathrm{C}$ oven for 48 hours to cure. When the samples were removed from the oven and trimmed for sectioning, the rolled film was clearly visible in the block face (Figure 2A). Depending on the area sectioned, a single block face could contain a single piece (Figure 2B) or multiple pieces of film (Figure 2C). Samples were sectioned using an ultramicrotome at a speed of $1.20 \mathrm{~mm} / \mathrm{s}$ and a feed of $100 \mathrm{~nm}$, placed onto 200 mesh copper TEM grids and imaged with a JEOL JEM 2100 at $200 \mathrm{kV}$.

After imaging the new samples and comparing them to those in Figure 1, the method described above appeared to prevent the edges of the film from being damaged or folding (Figure 3). Instead, both edges of the film remain flat against the resin (Figures 3B and C), allowing for analysis of the entire cross-section of the film. The ability to analyze the entire cross-section of these thin and flexible films can allow determination of composition, layering and other important features related to the synthesis and uniformity of these films. 
References:

[1] Y. Liu et al., Materials Characterization. 58 (2007) 666-669.

[2] J.C. Bravman and R. Sinclair, Journal of Electron Microscopy Techniques. 1 (2005) 53-61.

[3] G. Park, Surface and Coatings Technology. 115 (1999) 52-56.

[4] The authors would like to acknowledge Aparna Subramaniam for her support of this project, as well as the research team from Sun Chemical Corporation for providing the thin polymer films.

Figure 1

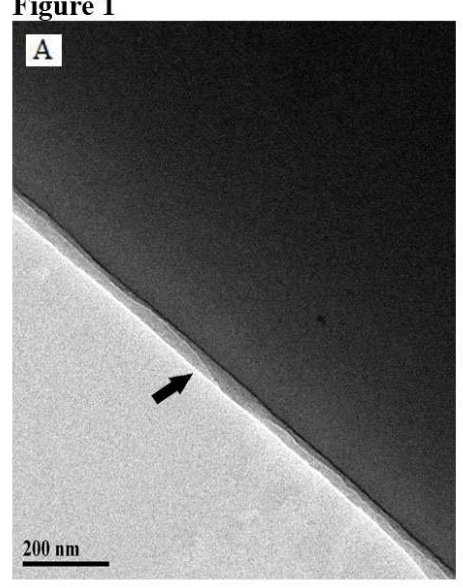

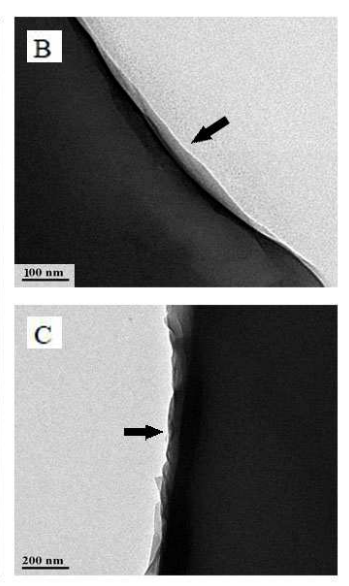

Figure 2
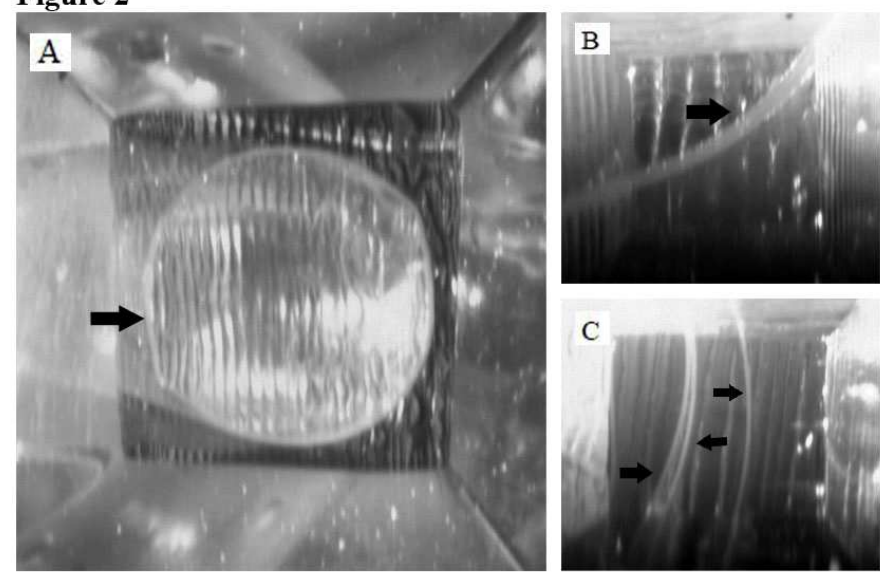

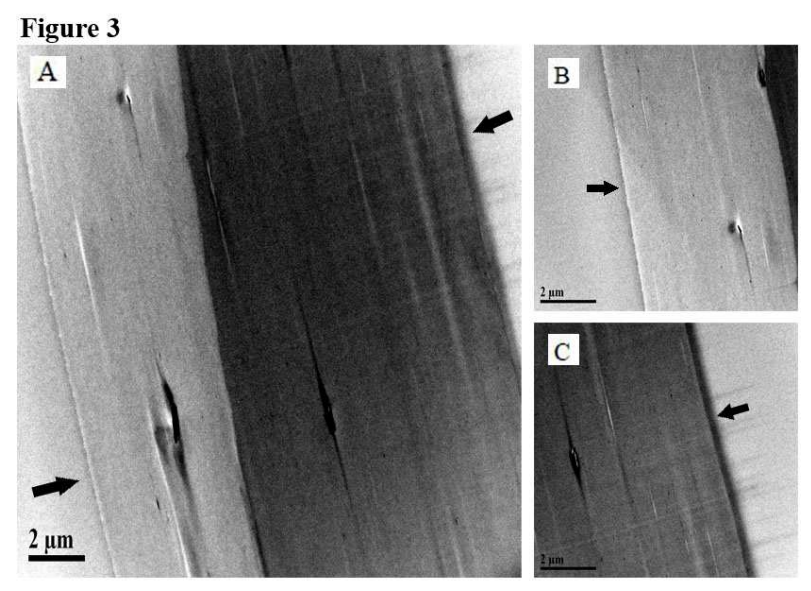

Figure 1: TEM images of film prepared using conventional techniques (dark area is film). (A\&B) Show folding that occurs at the edges of the film. (C) Shows damage that occurs due to the flexibility of the film when multiple strips are stacked. Arrows show areas of folding or damage.

Figure 2: Stereomicroscope images of trimmed resin blocks containing the rolled film. (A) An entire film tube in the block face. (B) Sample containing one film strip in the cutting window. (C) Sample containing 3 film strips in the cutting window. Arrows show film in the resin block.

Figure 3: TEM images of film after undergoing the described method. (A) Low magnification image of a film cross-section containing none of the damage found in Figure 1. (B\&C) Left and right edges of the film remaining attached to the resin with no damage. Arrows show boundary regions between the resin and the film. 